\title{
Totally drug-resistant tuberculosis strains: evidence of adaptation at the cellular level
}

\section{To the Editors:}

In the Eastern Mediterranean Region (EMRO), there are no accurate estimates of numbers of patients with multidrugresistant (MDR)/extensively drug-resistant (XDR) tuberculosis (TB). In 2006, we documented the existence and transmission of XDR-TB among patients with MDR-TB [1]. In a further study, we isolated more dangerous forms of bacilli, named totally drug-resistant (TDR) [2]. This group of strains showed in vitro resistance to all first- and second-line drugs tested (i.e. aminoglycosides, cyclic polypeptides, fluoroquinolones, thioamides, serine analogues and salicylic acid derivatives). TDRTB patients remained smear- and culture-positive after 18 months' median treatment with second-line drugs. Even changing the treatment to co-amoxyclav $(625 \mathrm{mg}$ per $8 \mathrm{~h})$ or clarithromycin $\left(1,000 \mathrm{mg} \cdot \mathrm{day}^{-1}\right)$, along with a high dose of isoniazid $\left(15 \mathrm{mg} \cdot \mathrm{kg}^{-1}\right)$ led to no improvement [2]. In the present study, transmission electron microscopy (TEM) was used to evaluate the differences between such deadly bacilli and MDR or susceptible ones at the ultrastructural level. Furthermore, to evaluate the genotyping patterns, the strains were subjected to spoligotyping and variable number of tandem repeats (VNTR) analysis.

Susceptibility testing against first- and second-line drugs was performed using the proportional method on LöwensteinJensen media [3].

For spoligotyping and VNTR analysis, five isolates from each group of strains (i.e., susceptible, MDR- and TDR-TB) were included in this study. The spoligotyping and VNTR analysis were performed on extracted DNA using standard protocols [4, 5].

For TEM analysis, a loop of Löwenstein-Jensen culture media was inoculated into Middlebrook 7H9 broth (Difco, Franklin Lakes, NJ, USA) supplemented with $0.2 \%$ glycerol and 10\% Middlebrook OADC enrichment (Difco). Cells at an optical density (OD) of 0.6 at $600 \mathrm{~nm}$ were used for further experiments. These cells were first centrifuged at $800 \times g$ for $5 \mathrm{~min}$, then the supernatant was adjusted to an OD of $580 \mathrm{~nm}$, corresponding to $6.3 \times 10^{7}$ colony-forming units of Mycobacterium tuberculosis per mL. $10 \mu \mathrm{L}$ of this supernatant were subjected to acid-fast bacilli and Gram staining. Nontuberculous mycobacterial contamination was check by culturing $100 \mu \mathrm{L}$ of supernatant on $7 \mathrm{H} 11$ agar plates. Niacin production, spoligotyping and IS6110-PCR were performed on isolated colonies from each plate. Thereafter, ultrathin sections for TEM analysis were prepared from fixed and solidified bacterial cells [6].

The identified spoligotypes were as follows: Haarlem I $(\mathrm{n}=3$; $20 \%)$; Beijing $(\mathrm{n}=2 ; 13 \%)$; EAI $(\mathrm{n}=4 ; 26 \%)$; CAS $(\mathrm{n}=4 ; 26 \%)$; $(\mathrm{n}=1 ; 6.6 \%)$; and $\mathrm{U}(\mathrm{n}=1 ; 6.6 \%)$ super families. The VNTR profiles of spoligotype strains were different in each superfamily.

In the TEM analysis, three different cell populations were observed at the exponential phase of TDR tubercle bacilli:
1) ordinary $(80-70 \%) ; 2)$ round or oval-shaped (15-20\%); and 3) extraordinarily thick cell wall bacilli $(21-26 \mathrm{~nm})$ that resembled features of stationary or anaerobic dormant bacilli (5-7\%) (fig. 1). These adapted forms were detected in all TDR isolates, irrespective of their superfamilies or genotypes patterns. The adapted forms of TDR tubercle bacilli were not detected in susceptible or MDR strains. Under the TEM, marked differences were observed in the thickness of cell walls: $20.2 \pm 1.5 \mathrm{~nm}$ and $17.1 \pm 1.03 \mathrm{~nm}$ for the TDR- and MDR-TB bacilli, respectively and $15.6 \pm 1.3 \mathrm{~nm}$ for the susceptible isolates $(\mathrm{p}<0.05)$.

While the problem of XDR-TB cases remains unresolved in much of the world, here we report on a more dangerous form of the disease, which we call TDR-TB [2]. In these strains, 30\% of the total $M$. tuberculosis populations transformed into adapted forms, producing either round or oval shaped bacilli (15-20\%), or having an extraordinarily thick cell wall (5-7\%) (fig. 1). These forms were not found among the susceptible or MDR population. At present, we have not demonstrated the transmission of TB disease by these round or oval-shaped bacilli, but the possibility of transmission cannot be ignored [7]. The TDR-TB cells with an extraordinarily thick cell wall $(26 \mathrm{~nm})$ resembled stationary or dormant-phase bacilli. Such cells were not found in longitudinal and cross-sectional studies at different levels of MDR and susceptible strains, although it has been reported that these cells $(1-3 \%)$ are present in normal population of $M$. tuberculosis [8]. Under normal circumstances, stationary cells are viable but non-dividing [8]. The TDR stationary bacilli in the process of division may represent the dominant population of TDR tubercle bacilli in the near future.

The next important issue is: what drugs can really penetrate these cells, which have cells walls about 21-26 nm thick? As such the tuberculosis chemotherapy is very different from that of other bacterial infections. Mycobacterium has different populations of bacilli which can survive in an aerobic environment, or low-PH conditions (the conditions necessary for pyrazinamide activity) and microaerophilic conditions [9]. Each of anti-TB drugs has a major role in dealing with one of these populations [9]. However, there are still persistent bacterial populations that are not killed by any available TB drugs [10]. Clinically, we have experienced such cases in our TB wards. These patients did not respond to any standardised treatment and remained smear- and culture-positive after prolonged therapy with second-line drugs. Therefore, the problem of TDR-TB should be taken very seriously and this is an issue requiring urgent attention from the global scientific community.

The genotyping results showed different VNTR profiles among identified superfamilies of M. tuberculosis (Haarlem, Beijing, EAI, CAS, and T). The obtained patterns ruled out recent transmission among the studied cases. TEM analysis showed no ultrastructural differences among identified superfamilies. Therefore, changes at the ultrastructural level of resistant 

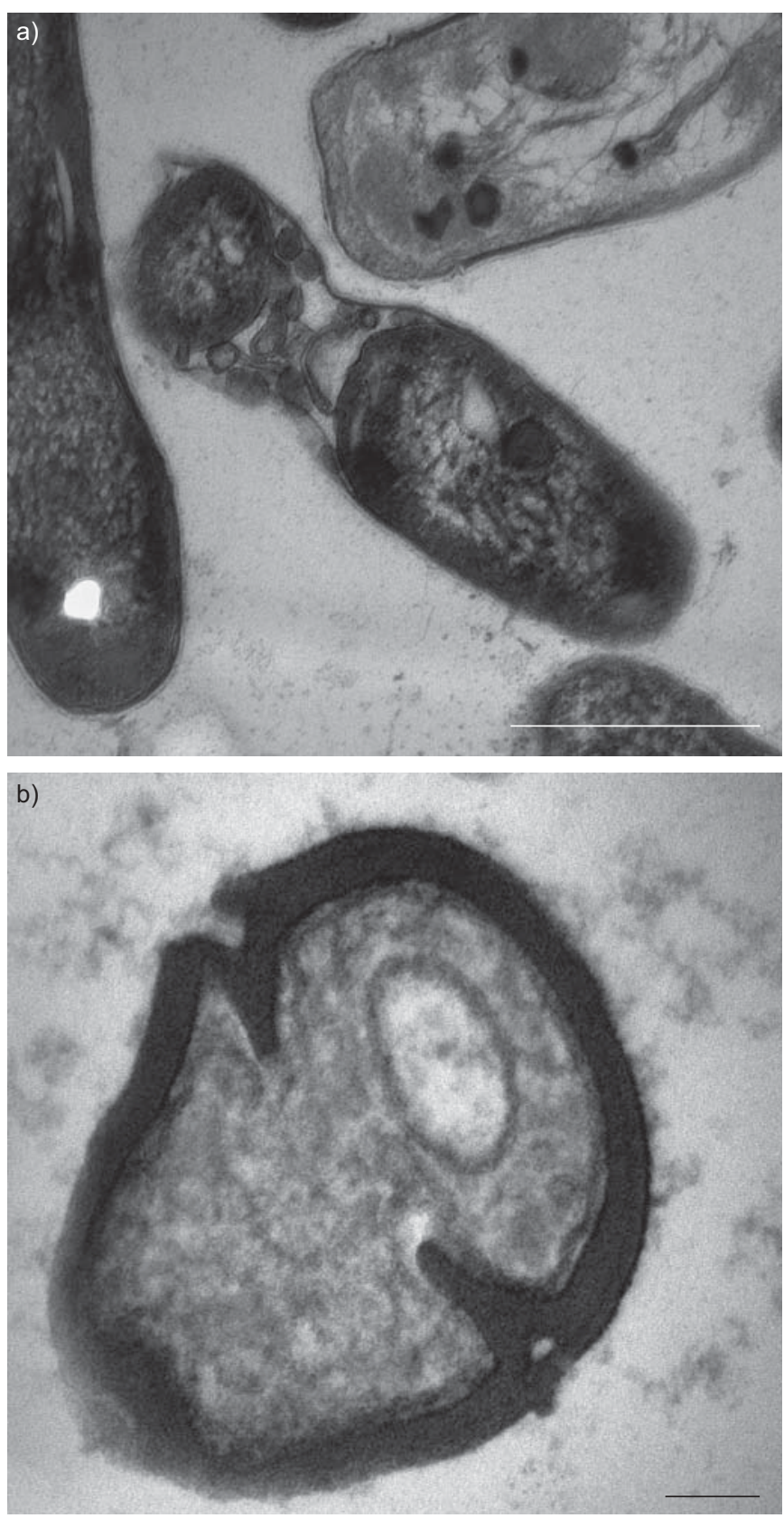

FIGURE 1. a) Totally drug-resistant (TDR)-tuberculosis (TB) bacilli with oval or round bodies inside them. Scale bar $=500 \mathrm{~nm}$. b) TDR-TB bacilli with stationary or anaerobic dormant bacilli in dividing stage. The increased size of the cell wall is very clear. Scale bar $=100 \mathrm{~nm}$.

strains may occur progressively and this is always preventable by prompt and reliable laboratory detection of drug-resistant TB cases.

A.A. Velayati*, P. Farnia*, M.R. Masjedi*, T.A. Ibrahim", P. Tabarsi*, R.Z. Haroun $^{\#}$, H.O. Kuan", J. Ghanavi ${ }^{\mp}$, P. Farnia and M. Varahram*

*Mycobacteriology Research Centre, and, "Experimental \& Animal Research Laboratory, National Research Institute of
Tuberculosis and Lung Disease, World Health Organization Collaborating Centre, Shahid Beheshti University (Medical Campus), Darabad, Tehran, Iran. "Microscopic unit, Institute of Bioscience, University Putra Malaysia, Serdang, Malaysia.

Correspondence: P. Farnia, Mycobacteriology Research Centre, National Research Institute of Tuberculosis and Lung Disease/ World Health Organization, Shahid Beheshti University (Medical Campus), Tehran, 19556, P.O. E-mail: pfarnia@hotmail.com

Support Statement: This study was sponsored by grant no: 0031/ A12/2007 from the Mycobacteriology Research Centre/National Research Institute of Tuberculosis and Lung Disease/World Health Organization.

Statement of Interest: None declared.

\section{ACKNOWLEDGEMENTS}

The authors wish to thank the staff of the Microscopy Unit at the Institute of Bioscience, University Putra Malaysia (Serdang, Malaysia). Also we sincerely thank the TB laboratory staff at the Mycobacteriology Research Centre/National Research Institute of Tuberculosis and Lung Disease (Tehran, Iran).

\section{REFERENCES}

1 Masjedi MR, Farnia P, Sorooch S, et al. Extensively drug-resistant tuberculosis: 2 years of surveillance in Iran. Clinical Infect Dis 2006; 43: 841-847.

2 Velayati AA, Masjedi MR, Farnia P, et al. Emergence of new forms of totally drug-resistant tuberculosis bacilli: super extensively drug-resistant tuberculosis or totally drug-resistant strains in Iran. Chest 2009; 136: 420-425.

3 World Health Organization (WHO). Guidelines for drug susceptibility testing for second line anti tuberculosis drugs for DOTs-plus WHO/CDC/TB/2001.288. Geneva, World Health Organization, 2001.

4 van Embden JD, Cave MD, Crawford JT, et al. Strain identification of Mycobacterium tuberculosis by DNA fingerprinting: recommendations for a standardized methodology. J Clin Microbiol 1993; 31 2406-2409.

5 Frothingham R, Meeker-O'Connell WA. Genetic diversity in the Mycobacterium tuberculosis complex based on variable numbers of tandem DNA repeats. Microbiology 1998; 144: 1189-1196.

6 Toda T, Takeya K, Koike M, et al. Electron microscopy of ultrathin sections of Mycobacterium. Fine structure of the cells grown in-vitro and in-vivo. Proc Japan Acad 1960; 36: 372-375.

7 Farnia P, Masjedi MR, Varahram M, et al. The recent-transmission of Mycobacterium tuberculosis strains among Iranian and Afghan relapse cases: a DNA-fingerprinting using RFLP and spoligotyping. BMC Infect Dis 2008; 8: 109-116.

8 Cunningham AF, Spreadbury CL. Mycobacterial stationary phase induced by low oxygen tension: cell wall thickening and localization of the 16-kilodalton $\alpha$-crystallin homolog. J Bacteriol 1998; 180: 801-808.

9 Mitchison DA. The action of antituberculosis drugs in short-course chemotherapy. Tubercle 1985; 66: 219-225.

10 Velayati AA, Farnia P, Masjedi MR, et al. Differences in cell wall thickness between resistant and non resistant of Mycobacterium tuberculosis; using transmission electron microcopy. J Chemotherapy 2009; 55: 303-307.

DOI: 10.1183/09031936.00081909 